\title{
Effective dimensions and percolation in hierarchically structured scale-free networks
}

\author{
Víctor M. Eguíluz, ${ }^{1}$ Emilio Hernández-García, ${ }^{1}$ Oreste Piro, ${ }^{1}$ and Konstantin Klemm ${ }^{2}$ \\ ${ }^{1}$ Instituto Mediterráneo de Estudios Avanzados IMEDEA (CSIC-UIB), E07071 Palma de Mallorca, Spain \\ ${ }^{2}$ Niels Bohr Institute, CATS, Blegdamsvej 17, DK-2100 Copenhagen Ø, Denmark
}

(Dated: February 2, 2008)

\begin{abstract}
We introduce appropriate definitions of dimensions in order to characterize the fractal properties of complex networks. We compute these dimensions in a hierarchically structured network of particular interest. In spite of the nontrivial character of this network that displays scale-free connectivity among other features, it turns out to be approximately one-dimensional. The dimensional characterization is in agreement with the results on statistics of site percolation and other dynamical processes implemented on such a network.
\end{abstract}

PACS numbers: 89.75.Hc, 05.10.-a

\section{INTRODUCTION.}

The most basic characteristic of any geometric structure is perhaps its dimensionality. The notion of dimension is intuitively associated with the amount of data necessary to locate a point on the structure, but the difficulties to formalize this association are known since more than a century ago [1]. A distinction should be made between definitions of dimensions based on topology and those based on measures and distances or metrics. While both types are important in different fields of science, the latter is very relevant in the description of fractal structures [2] and in dynamical systems theory [3].

Complex networks are a category of geometrical structures that has been thoroughly investigated in the last few years [4]. However, the possible characterization of complex networks in terms of suitably defined dimensions remains practically unexplored, with the exception of a few cases of networks constructed from or embedded in regular Euclidean lattices [5, 6]. This characterization should not only improve understanding of the different geometrical properties of various networks, but also clarify its impact on the dynamics of processes that might take place on them - percolation, disease propagation, information transmission, etc. Fractal dimensions, for example, might be useful to elucidate the connections between network topology correlations and dynamics which, apart from some isolated results [7], remain essentially not understood. Issues such as why two networks with the same degree distribution but different wiring details show different dynamical properties [8] are good candidates to be tackled with the tools of fractal geometry.

The aim of this Paper is to introduce a set of quantities, namely, the network dimensions with the purpose of providing a finer classification of networks with similar topological structure. As an application, we analyze a particular type of structured scale-free network [9, 10]. We show that some of its properties can be understood from the fact that it behaves close to one-dimensional with appropriately defined dimensions.

\section{DEFINITIONS.}

A network is a set of lines, the links, connecting points named nodes or sites. Topologically, any network is a one-dimensional object that by virtue of Whitney's theorem [1] can always be embedded in the three-dimensional Euclidian space. However, this topological dimension does not carry information related to the many interesting properties of networks. In a regular square lattice, for example, the number of sites within a given distance from a particular node asymptotically grows as the square of this distance. This example suggests that a metric definition of dimension could highlight better the two-dimensional character of the lattice than the topological dimension does.

A hierarchy of definitions for the dimension of a set that are associated to the properties of measures defined on that set were long ago introduced by Renyi [11], and have since been used with success in several fields. These dimensions were particularly helpful for the description of several natural fractal objects as well as for the characterization of chaotic trajectories in dynamical systems theory. In the standard definition the set to be characterized is first covered with a number $N(\epsilon)$ of boxes of size $\epsilon$. Let $\mu_{i}$ be the measure associated to the box $i$. Then, the spectrum of dimensions $\tilde{D}_{q}$ is defined by the scaling of the quantity $\Gamma(q, \epsilon) \equiv \sum_{i=1}^{N(\epsilon)} \mu_{i}^{q}$ for small $\epsilon$ :

$$
\tilde{D}_{q}=\lim _{\epsilon \rightarrow 0} \frac{1}{q-1} \frac{\ln \Gamma(q, \epsilon)}{\ln \epsilon} .
$$

$\tilde{D}_{0}, \tilde{D}_{1}$, and $\tilde{D}_{2}$ are the so-called capacity, information, and correlation dimensions respectively. It can be shown that $\tilde{D}_{q} \geq \tilde{D}_{q^{\prime}}$ if $q<q^{\prime}$. $\tilde{D}_{1}$ in Eq. (1) can in principle depend on the particular set of boxes covering the set which implies that an extremum requirement similar to the one in the original definition by Haussdorf [3] may be technically needed. Here we will postpone the consideration of these refinements to simplify implementation of practical numerical algorithms.

One natural way to define a measure on a network is to assign the unit of mass to each node. In this case, the measure of a portion of the network is the number of 
a)

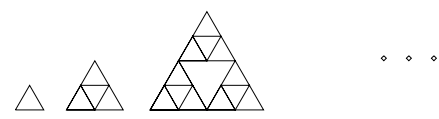

b)

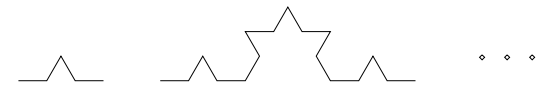

FIG. 1: a) The three initial steps in the construction of an infinite network of the Sierpińsky gasket type. The unit of length is kept to one link at each step of the construction, so that the relevant scaling is at large distances. b) The two initial steps in the construction of the nonbranching Koch-like network.

nodes that it contains. It is less easy to define a 'covering' of the network with boxes because it requires an a priori knowledge of the Euclidean space in which the network can be embedded. Since many networks are defined without referencing any embedding in a Euclidean space, alternative definitions based on intrinsic distances are necessary. In our context, a convenient distance $\operatorname{dist}(X, Y)$ between two nodes $X$ and $Y$ is the minimum number of links contained by a path connecting nodes $X$ and $Y$. This is a well-defined metric sometimes called chemical distance [12]. Distinctively from Euclidian and other distances commonly used to define dimensions, this one is integer valued. As a consequence, the scaling properties should be referred to the large distances limit instead of the opposite one usually considered.

A definition of the dimensions spectrum $D_{q}$, equivalent to Renyi's one (1) in those systems where both are applicable, is based on the scaling of the $q$-correlation function $C(q, L)[13]$. In a network of $M$ points, $C(q, L)$ is the number of $q$-tuplets of points in the network with mutual distances smaller than $L$, and divided by $M^{q}$. We then have:

$$
D_{q}=\lim _{L \rightarrow \infty} \frac{1}{q-1} \frac{\ln C(q, L)}{\ln L}
$$

The advantage of using (2) instead of (1) with the Euclidean distance replaced by the chemical one is that (2) does not require any box covering and therefore no a priori knowledge of the Euclidean embedding is necessary.

Finally, another equivalent definition [14] with easy practical implementation is the one based on the scaling of the number of neighbors within a given distance $L$ of a given site $X_{i}: \mu_{L}\left(X_{i}\right) \equiv(M-1)^{-1} \sum_{j \neq i} \Theta\left(L-\operatorname{dist}\left(X_{i}-\right.\right.$ $\left.X_{j}\right)$ ). $D_{q}$ is determined from moments of $\mu_{L}\left(X_{i}\right)$ :

$$
D_{q}=\lim _{L \rightarrow \infty} \frac{1}{q-1} \frac{\ln \left\langle\mu_{L}(X)^{q-1}\right\rangle_{X}}{\ln L}
$$

where the averages $\langle.\rangle_{X}$ are taken over all the nodes $X$

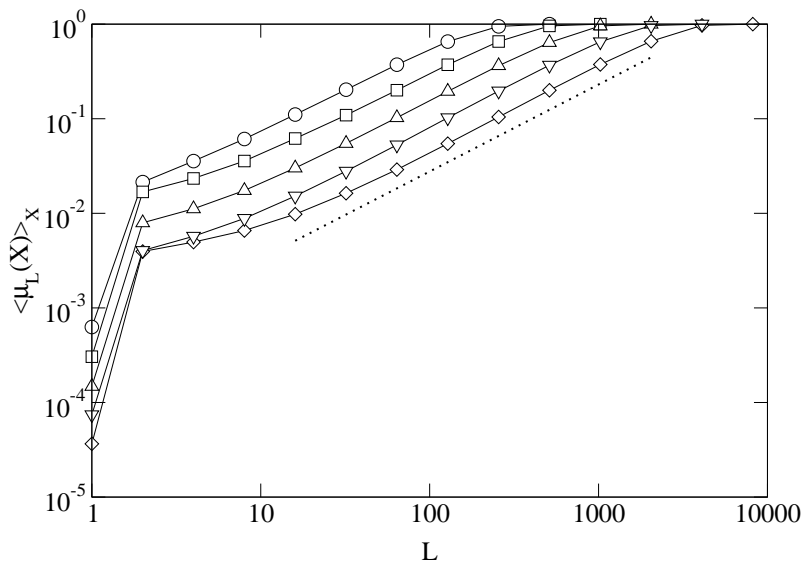

FIG. 2: Average number of nodes at distance $L$ from a given node. System sizes $10000,20000,40000,80000,160000$. The dashed line grows as $\sim L^{0.92}$. The averages have been done over 10 different networks and on each 1000 different starting nodes.

in the network. Application of l'Hôpital's rule gives

$$
D_{1}=\lim _{L \rightarrow \infty}\left\langle\frac{\ln \mu_{L}(X)}{\ln L}\right\rangle_{X} .
$$

Other equivalent definitions, based on the scaling of nearest-neighbor distances, or fixed mass methods 15], could be also implemented on networks, but we find definition (3) to be appropriate for our purposes. In cases in which an Euclidean distance can be defined, it can also be used in (2) or (3) to define quantities that would be denoted by low-case letters, $d_{q}$, to distinguish them from the case in which the chemical distance is used. The $d_{q}$ are essentially the classical fractal dimensions, but associated to the large-distance scaling.

It is instructive to calculate the dimension values for several simple networks. For example, for regular triangular, square, etc. lattices, it is easy to check that $D_{q}=2 \forall q . \quad D_{q}=3$ for the classical three-dimensional lattices (e.g. cubic, ...), etc. For a network with a star topology (i.e., a large number $N$ of nodes connected to a central hub), $D_{q}=\infty$, since all the nodes are at a finite distance (1 or 2 ) of each other even in the limit $N \rightarrow \infty$. The same happens for randomly wired networks. The usual implementation of the small world property, i.e. the introduction of links connecting arbitrarily remote nodes, leads also to $D_{q} \rightarrow \infty$. This has been explicitly demonstrated for a small-world model in [5], where effective dimensions essentially equivalent to $D_{2}$ are introduced and calculated as a function of spatial scales. At large scales ( $L \rightarrow \infty$ in our notation) a divergent quantity is obtained. Thus, it is useful to think of some of the characteristics of small worlds or random graphs as being associated to an infinite dimensionality. From the expressions in [16] or [17] one can also show that $D_{q}=\infty$ for the structures presented there.

After considering these examples, one may wonder if there is any non-regular complex network structure char- 


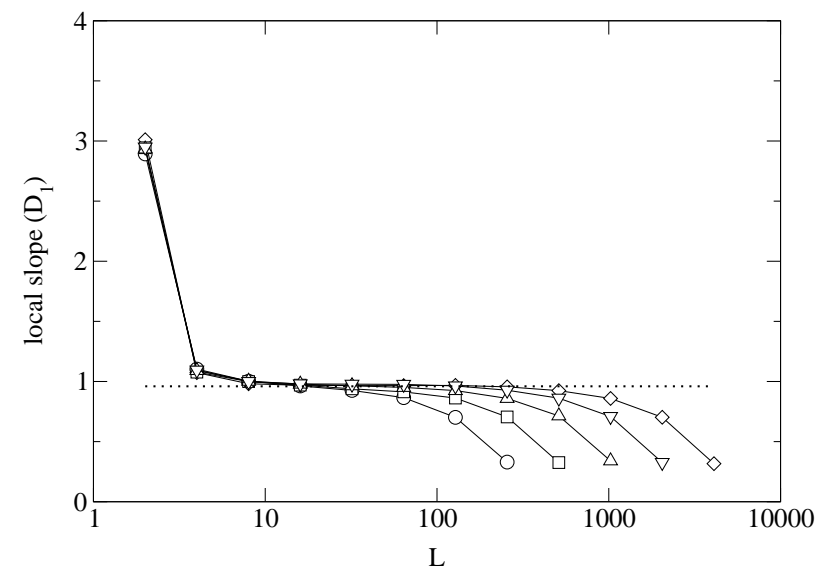

FIG. 3: Average local ratio $\left\langle\ln \left(\mu_{L}\right) / \ln L\right\rangle$. The plateau indicates a value $D_{1}=0.97$. Symbols and averaging as in Fig. 2

acterized by finite dimensions. We will show below that the answer is positive and that this finite dimensionality has dynamical consequences that distinguish processes occurring on these networks from the infinite-dimensional ones. Before that, we can mention that networks of arbitrary dimensionality can be constructed by following the rules used to construct recursively fractals of given dimension. For example, a classical fractal is the Sierpińsky gasket [2], constructed, generation after generation, by inscribing triangles inside the triangles originated in the previous generation. Since we are here characterizing large-scale features, it is better to consider the construction as the recursive joining of triangles to construct a larger and larger object (see Fig. [1). Since the resulting fractal structure is embedded in the plane, one can use the Euclidean distance and find as usual that $d_{q}=d_{0}=\ln 3 / \ln 2, \forall q$. If we use instead the chemical distance, with the lines in Fig. 10 identified as links of unit chemical length, we have also $D_{q}=d_{q}=\ln 3 / \ln 2, \forall q$. The situation is rather different for non-branching fractal constructions. For example, the classical Koch curve (Fig. П) has $d_{q}=\ln 4 / \ln 3$. But in terms of the chemical metrics, $\operatorname{dist}(X, Y)$, the distance between two points is always the number of nodes in between along the curve, so that we have $D_{q}=1 \forall q$, as for any other non-branching structure. In general, for networks constructed from the node and link structure of a classical fractal object, one expects $D_{q} \leq d_{q}$ since the Euclidian distance is in general shorter than the chemical one. This generally leads to finite chemical dimensions. In other constructions, the inequality may be reversed [6]. This last reference also provides an additional example of a network with finite dimensionality (in this case embedded in a Euclidean lattice).

More interesting is the fact that one can construct networks with finite dimensionality without any reference to fractal objects nor to Euclidean embedding spaces. We show here that the networks introduced by Klemm and Eguíluz [9, 10] are of such kind. This helps to understand

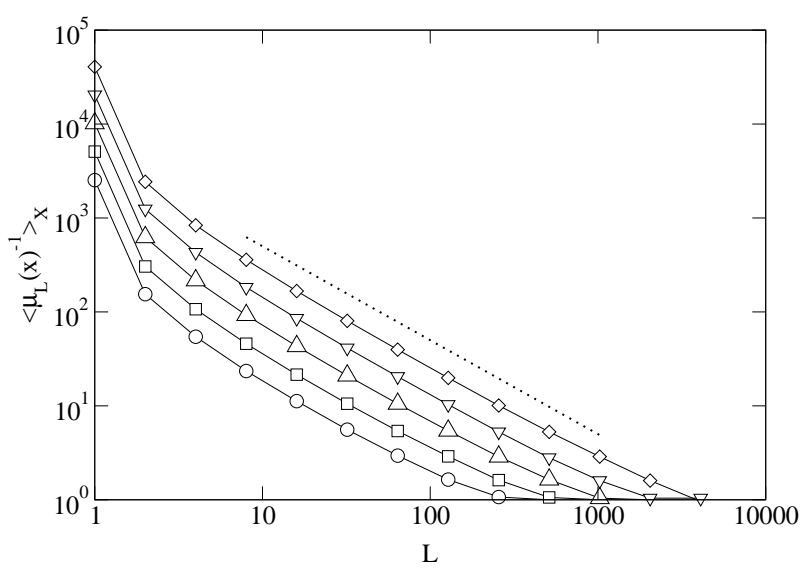

FIG. 4: Average $\left\langle\ln \left(\mu_{L}^{-1}\right)\right\rangle$. the dashed line corresponds to a function $\sim L^{-1}$. Symbols and averaging as in Fig. 2

the distinct behavior of these structures as compared to other considered in the literature.

\section{DIMENSION ANALYSIS OF THE KLEMM-EGUÍLUZ (KE) NETWORK.}

We have performed extensive numerical studies on the KE structured scale-free networks [9]. The procedure to generate them is as follows. Start with $m$ active, fullyconnected nodes. At each time step add a new node and attach $m$ new links between the new node and the old active nodes. Activate the new node and deactivate one of the active nodes according to the probability $\Pi(k) \propto$ $k^{-1}$, where $k$ is the degree of the node. This algorithm generates networks with a power law degree distribution $P(k) \sim k^{-\gamma}$, where $\gamma \simeq 3-1 / m$ [19]. In Fig. 2] we plot the average normalized number of nodes at distance $L,\left\langle\mu_{L}\left(X_{i}\right)\right\rangle$ for different system sizes, with $m=3$. By using (3) we find that the best fit gives the correlation dimension $D_{2}=0.92$. In Fig. 3 we plot the average slope $\left\langle\ln \mu_{L}\left(X_{i}\right) / \ln L\right\rangle$ for different system sizes. From (4) the plateau regime indicates an average value of the information dimension $D_{1}=0.97$. Finally from Fig. [4 the best fit gives, via (3), the capacity dimension $D_{0}=$ 1.0 .

The differences in the values of $D_{q}$ for different $q$ are a consequence of the inhomogeneity of the network. We will not concentrate here on this interesting point, but focus on the fact that the dimension estimates are finite and close to 1 . This confirms the conclusions in 10, 18], obtained from the scaling of the network's diameter, and opens the way for future characterization of the whole spectrum of dimensions in this and other network models. We can say that the network behaves, in the sense of the definitions introduced above, very close to onedimensional. It should be stressed that a priori there has not been an obvious Euclidian space containing the structure from which to calculate the dimensions $d_{q}$. In 


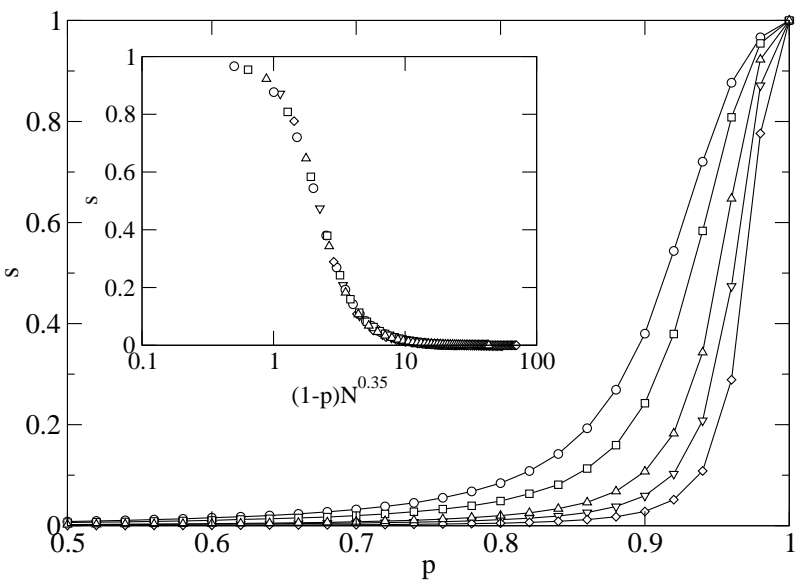

FIG. 5: Average relative size of the largest cluster $s$ in site percolation for different occupation probabilities $p$ in $m=3 \mathrm{KE}$ networks. The inset shows a finite size scaling $s=F\left((1-p) N^{0.35}\right)$. The average values have been obtained from 1000 percolation realizations in 10 different networks. Network size $N=10000$ (circles), 20000 (squares), 50000 (triangle up), 100000 (triangle down), 200000 (diamonds).

the following we consider dynamical processes occurring in $\mathrm{KE}$ networks and interpret them to the light of the dimension study.

\section{SITE PERCOLATION.}

An important process occurring on real networks is the propagation of information, or of diseases. If the nodes of the network are either susceptible to the disease with probability $p$ or immune to it with probability $1-p$, and the disease propagates through the links, the maximum number of individuals that can be affected by an epidemic outbreak is given by the size of the largest connected cluster in site percolation, where occupation of a site means susceptibility to the disease. We have performed numerical simulations of such a process in $\mathrm{KE}$ networks. In Fig. 5 we show the average relative size $s$ of the connected cluster around an occupied site for different system sizes and $m=3$. The results indicate that for any system size $N$ there is a (broad) transition to the percolating state at some value of the occupation probability $p_{c}$. However it is seen that $p_{c} \rightarrow 1$ as $N \rightarrow \infty$. Thus, the percolating transition occurs at $p_{c}=1$ in the infinite-size limit. This is precisely the expected behavior in a one-dimensional structure. The inset shows that the relative size of the largest cluster scales as $F\left((1-p) N^{\alpha}\right)$, where the exponent $\alpha$ depends on the average connectivity of the network. This finite size scaling behavior is also what one finds for percolation in one-dimensional regular lattices. In addition we have found that $\alpha=0.35$ for $m=3$, while $\alpha \simeq 0.21$ for $m=5$, suggesting that $\alpha$ is a decreasing function of the average degree $\langle k\rangle=2 \mathrm{~m}$. These numbers are in good agreement with site percolation in one-dimensional lattices with radius of interaction $z$ (i.e. two occupied sites are considered connected if they are $z$ or less sites apart, so that the degree of each site is $2 z)$. There one can show that $\alpha=z^{-1}$.

It is worth noting that this result is in contrast with the zero percolation threshold found [20] in random scalefree networks of the Barabási-Albert type [21]. For such networks, we have checked (for $q=0,1,2$ ) that the moments $\left\langle\mu^{q}\right\rangle$ do not scale as a power law of $L$, but instead $D_{q} \rightarrow \infty$ in this case. Thus, the different behavior may be associated to the different dimensionality.

\section{CONCLUSIONS.}

We have introduced definitions of dimensions useful for the study of complex networks. In particular, we have calculated the capacity, information and correlation dimension of a type of hierarchically structured scale-free network. We have shown that some dynamical properties of this class of networks can be understood in the light of the dimension analysis. It would be worth to search for other complex networks displaying finite dimension spectra. We think that possible candidates would be those with some underlying "regular" topology [6, 22].

We acknowledge financial support from MCyT (Spain) and FEDER (EU) through Projects CONOCE BFM2000-1108 and BFM2002-04474-C02-01.
[1] See for example the Appendix B in E.A. Jackson, Perspectives of Nonlinear Dynamics, Vol. 1 (Cambridge University Press, Cambridge, 1989) for a summary on the most important definitions.

[2] B.B. Mandelbrot, Fractals: Form, Chance, and Dimension, (Freeman, San Francisco, 1977).

[3] E. Ott, Chaos in Dynamical Systems (Cambridge University Press, Cambridge, 1993).

[4] R. Albert, A.-L. Barabási, Rev. Mod. Phys. 74, 47 (2002); S. N. Dorogovtsev, J. F. F. Mendes, Adv. Phys. 51, 1079 (2002).
[5] M.E.J. Newman, D.J. Watts, Phys. Rev. E 60, 7332 (1999).

[6] A.F. Rozenfeld, R. Cohen, D. ben-Avraham, S. Havlin, Phys. Rev. Lett. 89, 218701 (2002).

[7] M. Boguñá, R. Pastor-Satorras, A. Vespignani, cond-mat/0208163

[8] V.M. Eguíluz, K. Klemm, Phys. Rev. Lett. 89, 108701 (2002).

[9] K. Klemm, V.M. Eguíluz, Phys. Rev. E 65, 036123 (2002).

[10] K. Klemm, V.M. Eguíluz, Phys. Rev. E 65, 057102 
(2002).

[11] A. Rényi, Probability Theory (North Holland, Amsterdam, 1970).

[12] S. Havlin, in Kinetics of Aggregation and Gelation, ed. by F. Family and D.P. Landau (Elsevier, Amsterdam, 1984).

[13] H.G.E. Hentschel, I. Procaccia, Physica D 8, 435 (1983).

[14] R. Benzi, G. Paladin, G. Parisi, A. Vulpiani, J. Phys. A 17, 3521 (1984).

[15] W. van de Water, P. Schram, Phys. Rev. A 37, 3118 (1988); M. de Rover, W. van de Water, Phys. Rev. E 51, 4132 (1995).

[16] S.N. Dorogovtsev, A.V. Goltsev, J.F.F. Mendes, Phys.
Rev. E 65, 066122 (2002).

[17] S. Jung, S. Kim, B. Kahng, Phys. Rev. E 65, 056101 (2002).

[18] A. Vázquez, M. Boguñá, Y. Moreno, R. Pastor-Satorras, A. Vespignani, cond-mat/0209183

[19] K. Klemm, PhD thesis, Niels Bohr Institute, University of Copenhagen (2003)

[20] R. Pastor-Satorras, A. Vespignani, Phys. Rev. Lett. 86, 3200 (2001).

[21] A.-L. Barabási, R. Albert, Science 286, 509 (1999).

[22] C.P. Warren, L.M. Sander, I.M. Sokolov, Phys. Rev. E 66, 056105 (2002). 